\title{
A Description of the Initial Fire Sprinkler Spray
}

\author{
T. M. Myers, A. W. Marshall \\ Department of Fire Protection Engineering, University of Maryland, College Park, MD \\ 20742, USA
}

\begin{abstract}
Accurate representation of the fire sprinkler spray enables quantitative engineering analysis of fire suppression performance. Increasingly, fire sprinkler systems are analyzed with computational fluid dynamics (CFD) fire models where the sprinkler spray is simulated with Lagrangian particles dispersed throughout the fire induced flow. However, there is limited guidance for representing the complex, spatio-stochastic characteristics of the initial sprinkler sprays in terms of these Lagrangian particles. The present work establishes a descriptive analytical framework for the initial sprinkler spray that is rigorously grounded in statistical theory, related to local spray properties, and capable of translating high-fidelity measurements into CFD inputs. This framework describes the initial sprinkler spray as a unified probability distribution function, varying over an initialization surface, and statistically representing measurements of near field local spray properties (volume flux, drop size distribution, and drop sizevelocity correlation). Lagrangian particles accurately representing the sprinkler spray may be initialized by a stochastic sampling of this probability distribution function. This novel representation enables high-fidelity initialization of the sprinkler spray in CFD fire models, improving their utility in quantitative engineering analysis.
\end{abstract}

Keywords: fire sprinkler; spray modeling; atomization; suppression; spray theory; drop distribution function; Lagrangian particle; fire model; computational fluid dynamics; CFD

Preprint submitted to Fire Safety Journal

April 28, 2016

(C) 2016. This manuscript version is made available under the Elsevier user license http://www.elsevier.com/open-access/userlicense/1.0/ 


\section{Nomenclature}

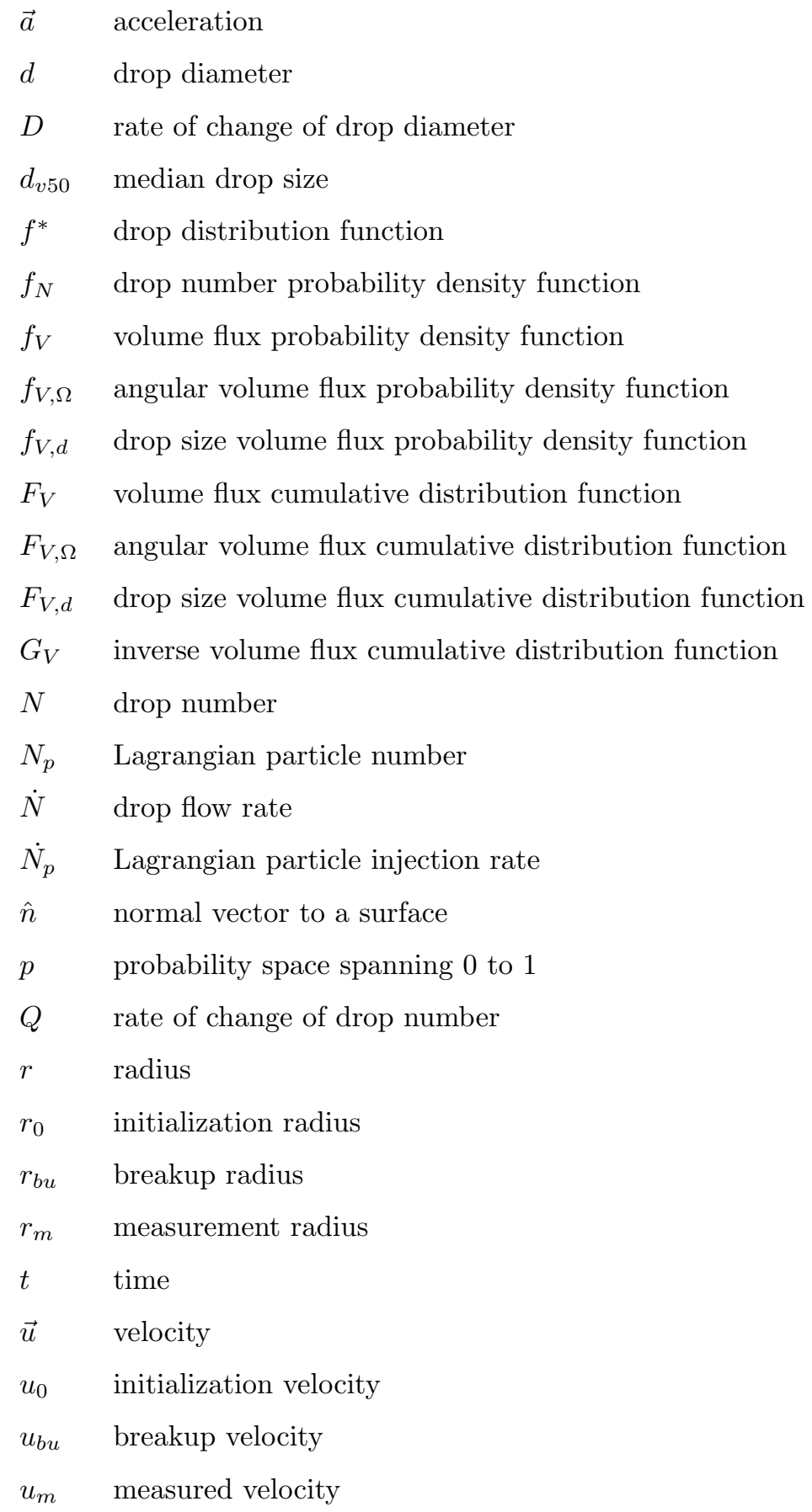




\title{
Nomenclature (cont.)
}

\author{
$\dot{V} \quad$ volume flow \\ $\dot{V}^{\prime \prime} \quad$ volume flux \\ $W_{p}$ particle weighting factor \\ $\vec{x} \quad$ position \\ Greek Symbols \\ $\Gamma \quad$ drop size distribution width \\ $\theta \quad$ elevation angle \\ $\psi \quad$ azimuthal angle \\ $\Omega$ solid angle
}

\section{Introduction}

Fire sprinklers provide a simple and cost effective spray dispersion method to arrest fire growth 11. A typical pendent sprinkler, as seen in Fig. 1 , consists of a thermal activation element and flow controlling elements; specifically, the

5 orifice, frame arms, boss, and deflector. Upon activation, a water jet (created by the orifice) strikes the frame arms, boss, and deflector, transforming the jet into a complex, cascading series of sheets, jets, and drops [2]. These sheets and jets move outward away from the sprinkler, interact with the surrounding air, and, because of aerodynamic instabilities, eventually break into drops. These drops form a dilute spray flying outward; dispersing through combustion products, plume, flame, and reactants; and interacting with surfaces.

The jet impingement atomization method produces a highly non-uniform spray, consistent with measurements by Sheppard [3, 4. Recent work by Ren [5] introduced a method characterizing measurements of the near-field fire sprinkler 15 spray in support of numerical simulation of sprinkler spray dispersion. These measurements, as well as detailed measurements by Zhou [6], reveal large elevation and azimuthal variations in near-field volume flux, drop size, and velocity corresponding to geometric features of the fire sprinkler head. An example typifying these spatial variations is seen in Fig. 2, a measurement of the near-field 

sity of Maryland's spray lab with the Spatially-resolved Spray Scanning System (4S) 7]. Any description of the fire sprinkler spray used for high fidelity fire protection analysis must capture these spatial variations.

Increasingly, computational fluid dynamics (CFD) fire models, like the Fire decisions [8, 9]. Both FDS and FireFOAM use an Eulerian-Lagrangian (EL) approach to describe multiphase flows. In the EL approach, the continuous phase (i.e. the gas phase, including the combustion products, plume, flame, and reactants) is represented as an evolving Eulerian field, while the dispersed [10]. In these models, Lagrangian particles, representing the sprinkler spray, are injected into the modeled domain. Particle motion is determined by solving the Lagrangian equations of motion and interaction with the Eulerian continuous phase is handled through a variety of sub-models (e.g. heat transfer, drop

There is limited guidance as to how the complex, spatio-stochastic characteristics of the initial fire sprinkler sprays can be represented in terms of these Lagrangian particles. Modeling is further complicated by the limited ability of current fire models to predict fire sprinkler atomization [6]. As a result, the initial fire sprinkler spray must be specified from measurements. Work by Ren [5] successfully identified the importance of measured local spray properties, but did not fully connect these filtered measurements to a robust statistical description of the flow or provide guidance to their implementation in high fidelity Lagrangian particle spray dispersion simulations.

45 statistical theory, tied to near-field measurements, and suitable for translating these measurements into a Lagrangian spray modeling framework. First, the drop distribution function is introduced, providing a rigorous statistical description for any dilute spray. This drop distribution function is then applied 50 
probability density function varying across an initialization surface. This probability density function, and the corresponding cumulative distribution function are related to locally measured spray properties. The net result of the current study is an initial fire sprinkler spray description scheme, for use in measurement and modeling, presented with requisite theoretical basis, implementation framework, and CFD application guidance.

\section{Theory}

A dilute spray is stochastic and locally unsteady. Despite this behavior, at sufficiently large spatial and temporal scales, a spray may be statistically described by a probability distribution function, $f^{*}$, called the drop distribution function [11]. This distribution function is defined such that the number of drops, $N$, in a phase space is assumed to be quasi-steady with time and is given as

$$
\int_{[\vec{x}, \vec{u}, d]} f^{*}(\vec{x}, \vec{u}, d) d \vec{x} d \vec{u} d d=N
$$

where the phase space spans the volume space, $\vec{x}$, the velocity space, $\vec{u}$, and the drop diameter space, $d$. For convenience, the phase space over which the drop distribution function exists may vary, and is denoted by the variables in parenthesis. As a result, the dimensions of $f^{*}$ may vary depending on the defined phase space.

The drop distribution function description of a spray has the advantage of being conceptually consistent with the way dilute sprays are measured, where the statistical properties of the spray are determined by measuring the spray in a limited volume of space over a sufficiently long period of time.

The evolution equation for the drop distribution function, called the spray equation, is given as

$$
\frac{\partial f^{*}}{\partial t}=-\frac{\partial}{\partial d}\left(D f^{*}\right)-\overrightarrow{\nabla_{x}} \cdot\left(\vec{u} f^{*}\right)-\overrightarrow{\nabla_{u}} \cdot\left(\vec{a} f^{*}\right)+Q
$$


where

$$
\begin{aligned}
& \vec{u} \equiv \frac{d \vec{x}}{d t}, \\
& \vec{a} \equiv \frac{d \vec{u}}{d t}, \\
& D \equiv \frac{d d}{d t},
\end{aligned}
$$

and $Q$ accounts for the formation of new drops in a non-colliding spray.

The direct solution of the spray equation, Eq. 2, is non-trivial and computationally expensive with traditional finite-volume or finite difference techniques [12, 13. Instead, an alternative solution based on Lagrangian particle methods

80 is commonly used to approximate the solution to Eq. 2.

In the Lagrangian particle method, the flow is seeded with $N_{p}$ Lagrangian particles chosen represent the drop distribution function. Each computational particle may represent many drops, having an associated position vector, $\vec{x}$, velocity vector, $\vec{u}$, diameter $d$, and particle weighting factor, $W$, corresponding to the number of drop represented. These particles are, in effect, a statistical sampling of the drop distribution function. These $N_{p}$ particles may then evolve individually according to Eqs. 3,5, indirectly satisfying the spray equation, Eq. 2.

Lagrangian particle evolution methods are well developed and well understood [12, 13]. The challenge for fire sprinkler sprays is describing the strong spatial variations measured in the initial spray and initializing Lagrangian particles to accurately represent these measurements.

\section{Initial Spray Description}

Because the state of the art in sprinkler sprays does not yet allow for prediction of atomization, initial sprays are generally described through a set of measured spray properties: volume flux, drop size, and velocity. Further, because 
strong spatial variations exist in typical sprinkler sprays, these spray properties must be measured locally. These local spray properties can be related to the probability density function, $f$, to completely describe the spray.

The probability density function is directly related drop distribution function, Eq1 by

$$
f_{N}(\vec{x}, \vec{u}, d)=f^{*}(\vec{x}, \vec{u}, d) / N,
$$

where $f_{N}$ describes the number fraction of drops in the multivariate phase space. The drop number probability density function, $f_{N}$, has the property

$$
\int_{[\vec{x}, \vec{u}, d]} f_{N}(\vec{x}, \vec{u}, d) d \vec{x} d \vec{u} d d=1 .
$$

The drop number probability density function, $f_{N}$, can be used to stochastically inject Lagrangian particles to represent measurements.

\subsection{Relationship to Measurement Topology}

To facilitate conversion between measurements and modeling, the probability density function should reflect measurement topology and be directly related to measured properties.

To minimize the influence of the gas phase on the spray, the spray is measured as close to the sprinkler head as possible. However, the spray can only be measured after complete atomization. Atomization occurs at a radius from the sprinkler head of between 0.1 and $0.4 \mathrm{~m}$ for most sprinklers [14. Further, experimental observations have shown that the spray, when very near the sprinkler head, tends to move radially outwards from the sprinkler head [5]. As a result, measurements are generally made along a spherical surface with its origin on the sprinkler head.

The probability density function, $f_{N}$, given in Eq. 7, may be redefined in spherical coordinates as

$$
\int_{[\vec{r}, \vec{u}, d]} f_{N}(\vec{r}, \theta, \phi, \vec{u}, d) r^{2} d r d \Omega d \vec{u} d d=1
$$


120 elevation angle, and $\phi$ is azimuthal angle, and where $d \Omega$ is the differential solid angle defined as

$$
d \Omega=\sin (\theta) d \theta d \phi
$$

For sprinkler sprays it is generally not the number of drops per unit volume but the volume flux at surfaces that governs sprinkler performance 1]. Keeping with this flux perspective, the drop distribution can be described in terms of a drop volume flux through a given surface (instead of in terms of a drop number in a volume) given by

$$
\int_{[r, \theta, \phi, \vec{u}, d]} f_{V}(r, \theta, \phi, \vec{u}, d) r(\theta, \phi)^{2} d r d \Omega d \vec{u} d d=1
$$

where

$$
f_{V}(r, \theta, \phi, \vec{u}, d)=f_{N}(r, \theta, \phi, \vec{u}, d)(\vec{u} \cdot \hat{n})(\pi / 6) d^{3} / \dot{V}
$$

$\hat{n}$ is the vector normal to the surface, and

$$
\dot{V}=\dot{N}\left(\pi d^{3} / 6\right)
$$

130

This function describes the density of water volume flux passing through a given angular position on the surface $r(\theta, \phi)$. While Eq. 10 is valid in general, the surface flux based formulation is especially well suited for describing the near-field spray characteristics on the surface where measurements are made, $r_{m}(\theta, \phi)$, and correspondingly where Lagrangian particles may be initialized with known characteristics.

\subsection{Relationship to Measured Properties}

The volume flux based probability density function, $f_{V}$, is chosen to represent the initial spray because it conveniently reflects measurement methods. What remains is to relate the measured properties to the probability density function. 
As previously mentioned in Sec. 3.1. a spherical coordinate system was chosen to describe the spray because observation of the spray reveals that all drops in the initial spray tend to move radially away from the sprinkler head [5]. Because the velocity vector of the initial spray is assumed to be directed 145 approximately radially out of the sprinkler head, it may be seen that

$$
\vec{u} \cdot \hat{n} \approx|\vec{u}|,
$$

where $\hat{n}$ is aligned with $\vec{r}$ at any given angular location. Additionally, drops of the same size tend to move at the same velocity, as seen in Fig. 3, which shows the drop size-velocity correlation for drops measured in the same pendent sprinkler as Fig. 2 at $\theta=115^{\circ}$ and $\phi=245^{\circ}$. As a result, drop velocity can be described as a characteristic velocity, $u_{0}(\theta, \psi, d)$.

If all drops are assumed to emerge from the surface $r_{0}(\theta, \psi)$ with an initial velocity, $u_{0}(\theta, \psi, d)$, the phase space of the volume flux probability density function, $f_{V}$, from Eq. 10, may be further restricted to vary only with angular location, $(\theta, \phi)$, and drop size, $d$, as

$$
\int_{[\theta, \phi, d]} f_{V}(\theta, \phi, d) r_{0}(\theta, \phi)^{2} d \Omega d d=1 .
$$

This probability density function may be decomposed into the conditional probabilities

$$
f_{V}(\theta, \phi, d)=f_{V, \Omega}(\theta, \phi) f_{V, d}(d \mid \theta, \phi),
$$

where $f_{V, \Omega}(\theta, \phi)$ is the probability of finding volume flux from any drops emerging from the angular coordinates $(\theta, \phi)$, and $f_{V, d}(d \mid \theta, \phi)$ is the probability of finding volume flux from drops of size $d$ given an angular location $(\theta, \phi)$.

To match the literature descriptions of drop size distributions and for convenience in initializing Lagrangian particles, the spray may also be described by a cumulative distribution function. The volume flux cumulative distribution function corresponding to the volume flux probability density function, $f_{V}$, is 
defined as

$$
F_{V}(\theta, \phi, d)=\int_{0}^{d} \int_{0}^{\phi} \int_{0}^{\theta} f_{V}(\theta, \phi, d) r_{0}(\theta, \phi)^{2} d \Omega d d,
$$

where $F_{V, \Omega}$ is the cumulative volume flow angular distribution, corresponding to the probability distribution function $f_{V, \Omega}$ and $F_{V, d}$ is the cumulative volume flow drop size distribution, corresponding to the probability distribution function $f_{V, d}$. The volume flow cumulative distribution function, $F_{V}(\theta, \phi, d)$, is constructed such that every phase space coordinate, $(\theta, \phi, d)$, corresponds to a unique value of $F_{V}$ between 0 and 1, which enables its later sampling to generate Lagrangian particles, as described in Sec. 4.

Both $F_{V, \Omega}$ and $F_{V, d}$ may be related to the measured volume flux and drop size, respectively, and the multiplied to provide a complete description of the flow, as per Eq. 17.

The cumulative volume flow angular distribution, $F_{V, \Omega}$, is related to the measured local volume flux normal to the initialization surface, $\dot{V}^{\prime \prime}(\theta, \phi)$, by Eq. 16 and

$$
f_{V, \Omega}(\theta, \phi)=\frac{\dot{V}^{\prime \prime}(\theta, \phi)}{\dot{V}} .
$$

The local volume flux of the initial spray may be obtained with spatially resolved measurements such as those measured with the University of Maryland's 4S, as seen in Fig. 2 and described by Jordan, et. al. 7 .

The cumulative drop size distribution, $F_{V, d}$, has been well explored in the literature and, for a typical fire sprinkler, can be well represented by a combination of log-normal and Rosin-Rammler distributions [15]. The cumulative volume fraction may be fit with two spatially varying parameters, median drop size, $d_{v 50}$, and drop size distribution width, $\Gamma$. This distribution may be written 


$$
F_{V, d}(d \mid \theta, \psi)= \begin{cases}\frac{1}{\sqrt{2 \pi}} \int_{0}^{d} \frac{\Gamma}{1.15 d^{\prime}} e^{\left(\frac{-\ln \left(\frac{d^{\prime}}{d_{v 55}}\right)^{2}}{2\left(\frac{1.15}{\Gamma}\right)^{2}}\right) d d^{\prime}} & d<d_{v 50}, \\ 1-e^{-0.693\left(\frac{d}{d_{v 50}}\right)^{\Gamma}} & d \geq d_{v 50}\end{cases}
$$

For typical sprinklers, $d_{v 50}$ varies between 0.5 and $3 \mathrm{~mm}$ and $\Gamma$ is between 2 and 3. Figure 4 shows $F_{V, d}$ and $f_{v, d}$ for the spray measured in the same pendent

185 and $\Gamma=2.2$.

The impact of spatial variations in drop size can be seen in Fig. 5, which shows the same spatially resolved volume flux from Fig. 2 decomposed into volume flux distributions from small drops $(d \leq 0.6 \mathrm{~mm})$ and large drops $(d>$ $0.6 \mathrm{~mm})$. The small and large drop spheres are calculated by multiplying the spatially resolved volume flux, $\dot{V}^{\prime \prime}(\theta, \phi)$, by $F_{V, d}(0.6 \mid \theta, \phi)$ and $1-F_{V, d}(0.6 \mid \theta, \phi)$, respectively. It becomes clear from this filtering that for this particular spray, the larger drops come primarily from the sprinkler tines, while smaller drops are produced uniformly across tines and slots.

The initial spray may thus be described by the volume flow cumulative volume fraction, $F_{V}(\theta, \phi, d)$, where $F_{V}$ is given, as in Eq. 15 by the product of $F_{V, \Omega}$ and $F_{V, d}$, given by Eqs. 18 and 19 , respectively, where all drops at a given angular location are assumed to originate from an initial radius $r_{0}(\theta, \phi)$, with a velocity magnitude, $u_{0}(\theta, \psi, d)$, and a velocity angle aligned with the radial direction at a given angular location. $F_{V}(\theta, \phi, d)$ provides a detailed description of the spray grounded in spray theory and measurements, and capable of use for initializing Lagrangian particles for the numerical simulation of spray evolution.

\section{Initializing Lagrangian Particles}

The stochastic nature of the spray makes it impossible to precisely simulate every drop in the sprinkler spray. Instead, Lagrangian particles reflecting the statistical properties of the spray are chosen. To capture measured properties the volume flow cumulative distribution function, $F_{V}$, is sampled. 
Every phase space coordinate, $(\theta, \phi, d)$, corresponds to a unique value of the volume flow cumulative distribution function, $F_{V}$ between 0 and 1 . Because $F_{V}$ is strictly increasing and continuous, an inverse distribution function or quantile function, $G_{V}$, may be defined as

$$
G_{V}(p)=\left(F_{V}\right)^{-1}(\theta, \psi, d),
$$

where $p$ spans 0 to 1 and each value of $p$ corresponds to a unique value of $\theta, \psi$, and $d$. No analytic solution is available for the volume flow cumulative distribution function, $F_{V}$, and so numerical methods must be used to calculate it's inverse, $G_{V}$.

To initialize $N_{p}$ Lagrangian particles, $N_{p}$ values of $p$ are selected randomly between 0 and 1. Each $p$ is used with the numerical solution to Eq. 20 to determine the particle's angular location, $\theta$ and $\psi$, and drop size, $d$. This numerical particle is then initialized at a radius $r_{0}(\theta, \psi)$ from the sprinkler head with a velocity $u_{0}(\theta, \psi, d)$. Each phase space location will be sampled with a frequency proportional to its volume flow, and thus each Lagrangian particle chosen in this manner must carry and equal volume. To conserve volume at each time step, a particle weighting factor, $W_{p}$, corresponding to the number of physical drops contained in each numerical drop is given by

$$
W_{p}=\frac{\dot{V} / \dot{N}}{\pi d^{3} / 6}
$$

where drops are assumed to be spherical.

In this way, Lagrangian particles are preferentially injected at the locations in phase space corresponding to the fraction of the total spray volume flow they carry. This method may be used with any particle injection rate, $\dot{N}_{p}$. Limited computational resources make it impractical to simulate the motion of every drop in the sprinkler spray, but improved accuracy is seen with increasing particle injection rate. The increase in accuracy results because selecting particles amounts to a statistical sampling of the specified mean properties of the spray, represented by $F_{V}$. The standard error of a sampled mean from the true mean 
is proportional to $1 / \sqrt{N}$ for normal distributions. This $1 / \sqrt{N}$ scale is thus a over a particular scale of interest by limited Lagrangian particles.

Initial spray particles from a pendent type sprinkler, using the measured values of volume flux, drop size, and drop velocity highlighted in Figs. 2 - 4 according to Eqs. 15 - 19, are displayed on the measurement surface in Fig. ${ }_{240}^{6} 6$. This group of 1000 Lagrangian particles passing through the measurement surface represents a statistical sample of the millions of drops generated by the spray every second, capturing the measured sprays complex phase space dependence with great fidelity. Fig. 6 shows regions of high particle density corresponding to the regions of high volume flux in Fig. 2. It is also clear that the drop size distribution presented in Fig. 4. The rays displayed in Fig. 6also reflect the volume weighted average local velocity based on measurement, but do not reflect the local detailed drop size - velocity correlation presented in Fig. 3. While this information is available from measurement, it is not convenient to include these correlations for each local measurement.

An alternative effective breakup surface, also derived from measurements, is included in Fig. 7 (a). This surface accounts for the local drop size velocity correlation with the added benefit of providing a facility to include the transfer of drop momentum to air (i.e. entrainment within the measurement surface). 255 The breakup surface is constructed from local drop size-velocity correlations recognizing from atomization theory 16 that drops have identical injection velocities at breakup. The effective local breakup radius (i.e. effective breakup surface) and initial velocities shown in Fig. 7 are determined from best-fits of these local drop size-velocity correlations. Figure 7(a) reveals a complex breakup surface consistent with qualitative images of the initial sprinkler spray. Figure 7(b) shows the same statistically sampled drops displayed in Fig. 6 but superimposed on the breakup surface. The rays show consistent drop velocities while variations in radial drop location are apparent. It is clear from Fig. 7(b) that larger drops are generated closer to the sprinkler where the sheets formed 

located radially outward where sheets are thinner.

\section{Conclusions}

The drop distribution function provides the mathematical foundation necessary to completely describe the complex spatio-stochastic characteristics of the sprinkler spray first explored by Ren [5]. This description of the spray is very general and provides unification between Lagrangian and Eulerian spray dispersion perspectives in a stochastic framework. Recent advances in spray measurement techniques applied to fire sprinklers can now produce detailed local volume fluxes, local drop size distributions, and local drop size-velocity ties on an initial surface, as presented in Eqs. 16,19 and directly connected to the drop distribution function, provides a mathematical grounding for their robust integration and interpretation in a broader physical context. The presented framework for the description of the initial spray provides useful insight when applied in the analysis of high fidelity spray measurements and is readily applied to stochastically generate an initial spray in modern detail rich CFD based fire models.

\section{Acknowledgements}

This project is supported by the U.S. National Science Foundation (NSF285 GOALI Award \#1236788). Discussions with Eric Link are gratefully acknowledged.

\section{References}

[1] G. Grant, J. Brenton, D. Drysdale, Fire suppression by water sprays, Prog. Energy Combust. Sci. 26 (2000) 79-130. 
[8] K. McGrattan, S. Hostikka, R. McDermott, J. Floyd, C. Weinschenk, K. Overholt, Fire Dynamics Simulator, Technical Reference Guide, Volume 1: Mathematical Model, Tech. rep., National Institute of Standards and [

[2] A. W. Marshall, Unraveling fire suppression sprays, in: Fire Safety Science, International Association for Fire Safety Science, 2011, pp. 61-75. doi: 10.3801/IAFSS.FSS.10-61.

[4] D. Sheppard, R. Lueptow, Characterization of Fire Sprinkler Sprays using Particle Image Velocimetry, Atomization and Sprays 15 (3) (2005) 341-362.

[5] N. Ren, H. R. Baum, A. W. Marshall, A comprehensive methodology for characterizing sprinkler sprays, Proceedings of the Combustion Institute 33 (2) (2011) 2547-2554. doi:10.1016/j.proci.2010.06.107.

[6] X. Zhou, S. P. D'Aniello, H. Z. Yu, Spray characterization measurements of a pendent fire sprinkler, Fire Safety Journal 54 (2012) 36-48. doi: $10.1016 / j . f$ iresaf.2012.07.007

[7] S. Jordan, A. Marshall, Spatially-Resolved Spray Measurements and their Implications, Fire Technology.

Technology, Gaithersburg, Maryland, USA (2013). doi:10.6028/NIST. SP. 1018-1.

URL http://www .thunderheadeng.com/wp-content/uploads/2013/08/

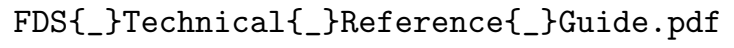

[9] FM Global, FireFOAM, available at: <https://github.com/ fireFoam-dev> (accessed: 11-30-2015).

[10] S. Subramaniam, LagrangianEulerian methods for multiphase flows, Progress in Energy and Combustion Science 39 (2-3) (2013) 215-245. 
doi:10.1016/j.pecs.2012.10.003

URL http://www.sciencedirect.com/science/article/pii/ S0360128512000603

[11] F. A. Williams, Spray Combustion and Atomization, Physics of Fluids 1 (6) (1958) 541. doi:10.1063/1.1724379

URL http://scitation.aip.org/content/aip/journal/pof1/1/6/10. $1063 / 1.1724379$

[12] S. Subramaniam, Statistical modeling of sprays using the droplet distribu325 tion function, Physics of Fluids 13 (3) (2001) 624-642. doi:10.1063/1. 1344893 .

[13] S. Balachandar, A scaling analysis for point-particle approaches to turbulent multiphase flows, International Journal of Multiphase Flow 35 (9) (2009) 801-810. doi:10.1016/j.ijmultiphaseflow.2009.02.013. URL http://dx.doi .org/10.1016/j.ijmultiphaseflow .2009.02.013

[14] N. Ren, a. W. Marshall, Characterizing the initial spray from large Weber number impinging jets, International Journal of Multiphase Flow 58 (2014) 205-213. doi:10.1016/j.ijmultiphaseflow.2012.08.004 URL http://dx.doi.org/10.1016/j.ijmultiphaseflow.2012.08.004

${ }_{335}$ [15] H. Yu, Investigation Of Spray Patterns Of Selected Sprinklers With The Fmrc Drop Size Measuring System, in: Fire Safety Science, Vol. 1, International Association for Fire Safety Science, 1986, pp. 1165-1176. doi: 10.3801/IAFSS.FSS.1-1165.

URL http://www . iafss . org/publications/fss/1/1165

340 [16] D. Wu, D. Guillemin, A. W. Marshall, A modeling basis for predicting the a initial sprinkler spray, Fire Safety Journal 42 (4) (2007) 283-294. doi: 10.1016/j.firesaf .2006.11.007.

URL http://dx.doi.org/10.1016/j.firesaf .2006.11.007 


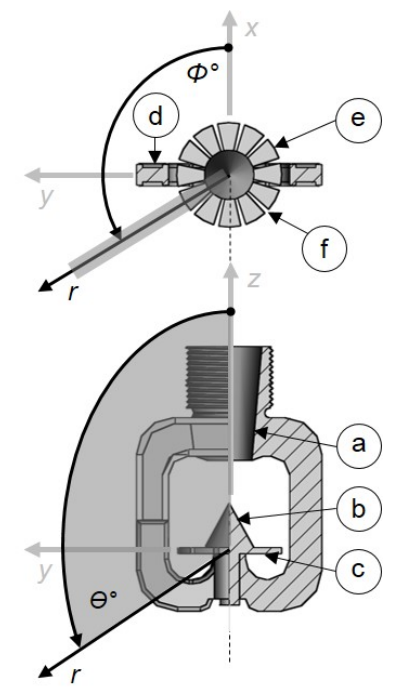

Figure 1: Partial cross section of a pendent sprinkler illustrating components; (a) orifice, (b) boss, (c) deflector, (d) frame arm (ref), (e) tines, and (f) slots [7.

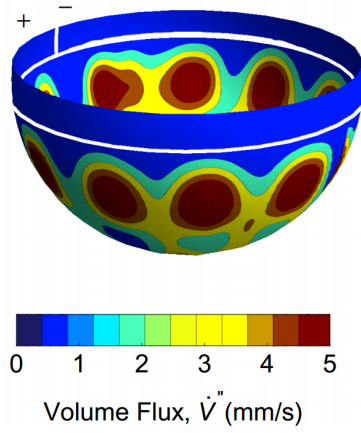

Figure 2: Highly non-uniform volume flux for a typical pendent sprinkler head, measured at a radius of $0.4 \mathrm{~m}$ from the sprinkler head [7]. 


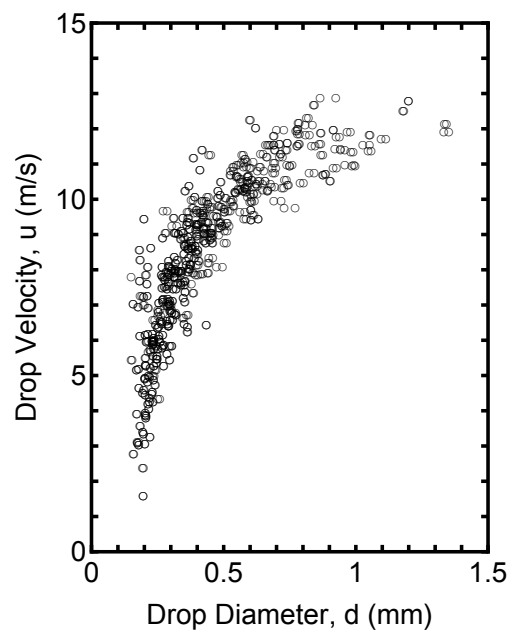

Figure 3: Local drop size-velocity correlation, $\vec{u}\left(115^{\circ}, 245^{\circ}, d\right)$ for the spray measured in the same pendent sprinkler as Fig. 2 . 


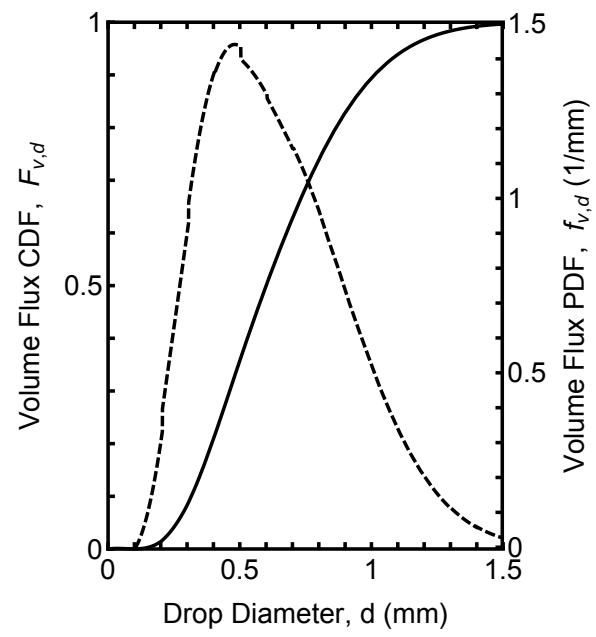

Figure 4: Cumulative volume fraction, $F_{v}\left(d, 115^{\circ}, 245^{\circ}\right)$, and spray probability density function, $f_{v}\left(d, 115^{\circ}, 245^{\circ}\right)$ with $d_{v 50}=0.57 \mathrm{~mm}$ and $\Gamma=2.2$.
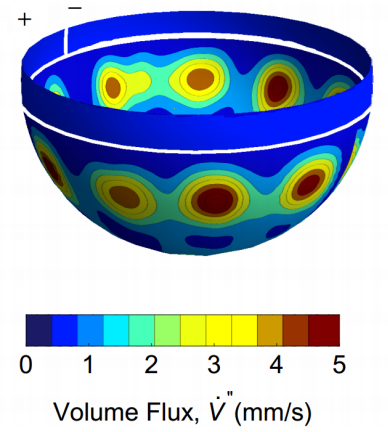
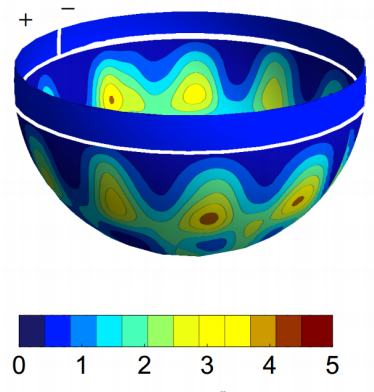

(b)

Figure 5: Volume flux from drops with diameter (a) greater than $0.6 \mathrm{~mm}$ and (b) less than 0.6 $\mathrm{mm}$ for a pendent type sprinkler head. The sum of these volume fluxes is the total volume flux, seen in Fig. 2 [7. 


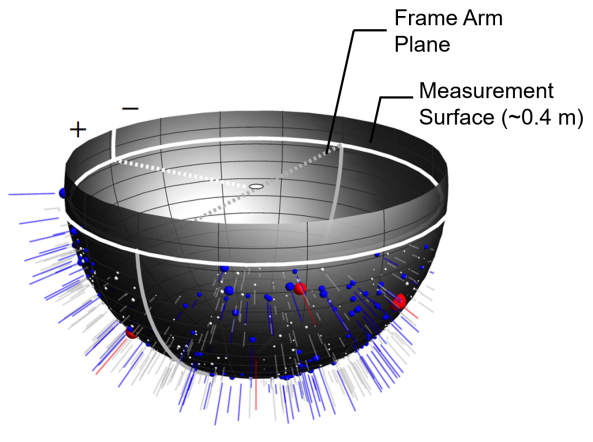

Figure 6: Lagrangian particles representing the initial spray; red $d>1.5 \mathrm{~mm}$, blue $0.5<d<$ $1.5 \mathrm{~mm}$, and white $d<0.5 \mathrm{~mm}$; and rays are scaled with velocity.

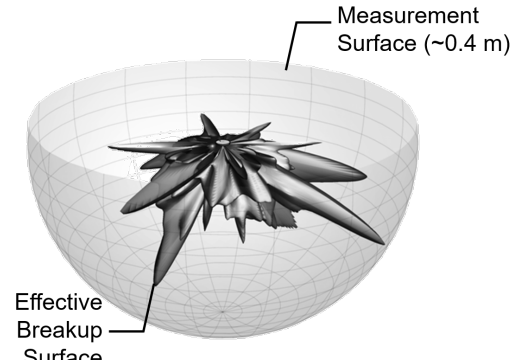

(b)

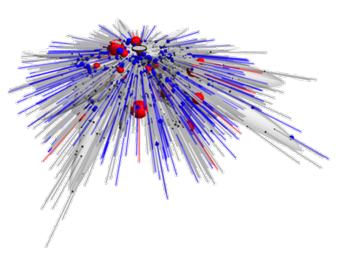

Figure 7: Effective sheet breakup surface (a) without Lagrangian particles and (b) with Lagrangian particles; red $d>1.5 \mathrm{~mm}$, blue $0.5<d<1.5 \mathrm{~mm}$, and white $d<0.5 \mathrm{~mm}$; and rays are scaled with velocity. 